\title{
GLOBAL EXISTENCE AND ENERGY DECAY OF SOLUTIONS TO THE CAUCHY PROBLEM FOR A WAVE EQUATION WITH A WEAKLY NONLINEAR DISSIPATION
}

\author{
ABBÈS BENAISSA AND SOUFIANE MOKEDDEM
}

Received 21 November 2003

We prove the global existence and study decay properties of the solutions to the wave equation with a weak nonlinear dissipative term by constructing a stable set in $H^{1}\left(\mathbb{R}^{n}\right)$.

\section{Introduction}

We consider the Cauchy problem for the nonlinear wave equation with a weak nonlinear dissipation and source terms of the type

$$
\begin{gathered}
u^{\prime \prime}-\Delta_{x} u+\lambda^{2}(x) u+\sigma(t) g\left(u^{\prime}\right)=|u|^{p-1} u \quad \text { in } \mathbb{R}^{n} \times[0,+\infty[ \\
u(x, 0)=u_{0}(x), \quad u^{\prime}(x, 0)=u_{1}(x) \quad \text { in } \mathbb{R}^{n}
\end{gathered}
$$

where $g: \mathbb{R} \rightarrow \mathbb{R}$ is a continuous nondecreasing function and $\lambda$ and $\sigma$ are positive functions.

When we have a bounded domain instead of $\mathbb{R}^{n}$, and for the case $g(x)=\delta x(\delta>0)$ (without the term $\lambda^{2}(x) u$ ), Ikehata and Suzuki [8] investigated the dynamics, they have shown that for sufficiently small initial data $\left(u_{0}, u_{1}\right)$, the trajectory $\left(u(t), u^{\prime}(t)\right)$ tends to $(0,0)$ in $H_{0}^{1}(\Omega) \times L^{2}(\Omega)$ as $t \rightarrow+\infty$. When $g(x)=\delta|x|^{m-1} x(m \geq 1, \lambda \equiv 0, \sigma \equiv 1)$, Georgiev and Todorova [4] introduced a new method and determined suitable relations between $m$ and $p$, for which there is global existence or alternatively finite-time blow up. Precisely they showed that the solutions continue to exist globally in time if $m \geq p$ and blow up in finite time if $m<p$ and the initial energy is sufficiently negative. This result was later generalized to an abstract setting by Levine and Serrin [12] and Levine et al. [11]. In these papers, the authors showed that no solution with negative initial energy can be extended on $[0, \infty[$, if the source term dominates over the damping term $(p>m)$. This generalization allowed them also to apply their result to quasilinear wave equations (see $[1,17])$. Quite recently, Ikehata [7] proved that a global solution exists with no relation between $p$ and $m$ by the use of a stable set method due to Sattinger [18].

For the Cauchy problem (1.1) with $\lambda \equiv 1$ and $\sigma \equiv 1$, when $g(x)=\delta|x|^{m-1} x(m \geq 1)$ Todorova [21] (see [16]) proved that the energy decay rate is $E(t) \leq(1+t)^{-(2-n(m-1)) /(m-1)}$ 
for $t \geq 0$. She used a general method introduced by Nakao [14] on condition that the data have compact support. Unfortunately, this method does not seem to be applicable in the case of more general functions $\lambda$ and $\sigma$.

Our purpose in this paper is to give a global solvability in the class $H^{1}$ and energy decay estimates of the solutions to the Cauchy problem (1.1) for a weak linear perturbation and a weak nonlinear dissipation.

We use a new method recently introduced by Martinez [13] (see also [2]) to study the decay rate of solutions to the wave equation $u^{\prime \prime}-\Delta_{x} u+g\left(u^{\prime}\right)=0$ in $\Omega \times \mathbb{R}^{+}$, where $\Omega$ is a bounded domain of $\mathbb{R}^{n}$. This method is based on a new integral inequality that generalizes a result of Haraux [6]. So we proceed with the argument combining the method in [13] with the concept of modified stable set on $H^{1}\left(\mathbb{R}^{n}\right)$. Here the modified stable set is the extended $\mathbb{R}^{n}$ version of Sattinger's stable set.

\section{Preliminaries and main results}

$\lambda(x), \sigma(t)$, and $g$ satisfy the following hypotheses.

(i) $\lambda(x)$ is a locally bounded measurable function defined on $\mathbb{R}^{n}$ and satisfies

$$
\lambda(x) \geq d(|x|)
$$

where $d$ is a decreasing function such that $\lim _{y \rightarrow \infty} d(y)=0$.

(ii) $\sigma: \mathbb{R}_{+} \rightarrow \mathbb{R}_{+}$is a nonincreasing function of class $C^{1}$ on $\mathbb{R}_{+}$.

Consider $g: \mathbb{R} \rightarrow \mathbb{R}$ a nondecreasing $C^{0}$ function and suppose that there exist $C_{i}>0$, $i=1,2,3,4$, such that

$$
\begin{gathered}
c_{3}^{\prime}|v|^{m} \leq|g(v)| \leq c_{4}^{\prime}|v|^{1 / m} \quad \text { if }|v| \leq 1, \\
c_{1}|v| \leq|g(v)| \leq c_{2}|v|^{r} \quad \forall|v| \geq 1,
\end{gathered}
$$

where $m \geq 1$ and $1 \leq r \leq(n+2) /(n-2)^{+}$.

We first state two well-known lemmas, and then we state and prove two other lemmas that will be needed later.

Lemma 2.1. Let $q$ be a number with $2 \leq q<+\infty(n=1,2)$ or $2 \leq q \leq 2 n /(n-2)(n \geq 3)$. Then there is a constant $c_{*}=c(q)$ such that

$$
\|u\|_{q} \leq \mathcal{c}_{*}\|u\|_{H^{1}\left(\mathbb{R}^{n}\right)} \quad \text { for } u \in H^{1}\left(\mathbb{R}^{n}\right)
$$

Lemma 2.2 (Gagliardo-Nirenberg). Let $1 \leq r<q \leq+\infty$ and $p \geq 2$. Then, the inequality

$$
\|u\|_{p} \leq C\left\|\nabla_{x}^{m} u\right\|_{2}^{\theta}\|u\|_{r}^{1-\theta} \quad \text { for } u \in \mathscr{D}\left((-\Delta)^{m / 2}\right) L^{r}
$$

holds with some constant $C>0$ and

$$
\theta=\left(\frac{1}{r}-\frac{1}{p}\right)\left(\frac{m}{n}+\frac{1}{r}-\frac{1}{2}\right)^{-1}
$$

provided that $0<\theta \leq 1$ (assuming that $0<\theta<1$ if $m-n / 2$ is a nonnegative integer). 
Lemma $2.3[10]$. Let $E: \mathbb{R}_{+} \rightarrow \mathbb{R}_{+}$be a nonincreasing function and assume that there are two constants $p \geq 1$ and $A>0$ such that

$$
\int_{S}^{+\infty} E^{(p+1) / 2}(t) d t \leq A E(S), \quad 0 \leq S<+\infty,
$$

then

$$
\begin{aligned}
& E(t) \leq c E(0)(1+t)^{-2 /(p-1)} \quad \forall t \geq 0, \text { if } p>1, \\
& E(t) \leq c E(0) e^{-\omega t} \quad \forall t \geq 0, \text { if } p=1,
\end{aligned}
$$

where $c$ and $\omega$ are positive constants independent of the initial energy $E(0)$.

Lemma 2.4 [13]. Let $E: \mathbb{R}_{+} \rightarrow \mathbb{R}_{+}$be a nonincreasing function and $\phi: \mathbb{R}_{+} \rightarrow \mathbb{R}_{+}$an increasing $C^{2}$ function such that

$$
\phi(0)=0, \quad \phi(t) \longrightarrow+\infty \quad \text { as } t \longrightarrow+\infty .
$$

Assume that there exist $p \geq 1$ and $A>0$ such that

$$
\int_{S}^{+\infty} E(t)^{(p+1) / 2}(t) \phi^{\prime}(t) d t \leq A E(S), \quad 0 \leq S<+\infty,
$$

then

$$
\begin{aligned}
& E(t) \leq c E(0)(1+\phi(t))^{-2 /(p-1)} \quad \forall t \geq 0, \text { if } p>1, \\
& E(t) \leq c E(0) e^{-\omega \phi(t)} \quad \forall t \geq 0, \text { if } p=1,
\end{aligned}
$$

where $c$ and $\omega$ are positive constants independent of the initial energy $E(0)$.

Proof of Lemma 2.4. Let $f: \mathbb{R}_{+} \rightarrow \mathbb{R}_{+}$be defined by $f(x):=E\left(\phi^{-1}(x)\right.$ ), (we remark that $\phi^{-1}$ has a sense by the hypotheses assumed on $\left.\phi\right) . f$ is nonincreasing, $f(0)=E(0)$, and if we set $x:=\phi(t)$, we obtain

$$
\begin{aligned}
\int_{\phi(S)}^{\phi(T)} f(x)^{(p+1) / 2} d x & =\int_{\phi(S)}^{\phi(T)} E\left(\phi^{-1}(x)\right)^{(p+1) / 2} d x=\int_{S}^{T} E(t)^{(p+1) / 2} \phi^{\prime}(t) d t \\
& \leq A E(S)=A f(\phi(S)), \quad 0 \leq S<T<+\infty .
\end{aligned}
$$

Setting $s:=\phi(S)$ and letting $T \rightarrow+\infty$, we deduce that

$$
\int_{s}^{+\infty} f(x)^{(p+1) / 2} d x \leq A f(s), \quad 0 \leq s<+\infty .
$$

Thanks to Lemma 2.3, we deduce the desired results.

Before stating the global existence theorem and decay property of problem (1.1), we will introduce the notion of the modified stable set. Let

$$
\begin{gathered}
K(u)=\left\|\nabla_{x} u\right\|_{2}^{2}+\|u\|_{2}^{2}-\|u\|_{p+1}^{p+1} \quad \text { if } \lambda \equiv 1, \\
I(u)=\left\|\nabla_{x} u\right\|_{2}^{2}-\|u\|_{p+1}^{p+1} \quad \text { if } \lambda \equiv \text { const }
\end{gathered}
$$


938 Cauchy problem for nonlinear wave equation

for $u \in H^{1}\left(\mathbb{R}^{n}\right)$. Then we define the modified stable set $\widetilde{\mathcal{W}}^{*}$ and $\widetilde{\mathscr{W}^{*} *}$ by

$$
\begin{aligned}
\widetilde{W}^{*} & \equiv\left\{u \in H^{1}\left(\mathbb{R}^{n}\right) \backslash K(u)>0\right\} \cup\{0\} \quad \text { if } \lambda \equiv 1, \\
\widetilde{W}^{* *} & \equiv\left\{u \in H^{1}\left(\mathbb{R}^{n}\right) \backslash I(u)>0\right\} \cup\{0\} \quad \text { if } \lambda \equiv \equiv \text { const. }
\end{aligned}
$$

Next, let $J(u)$ and $E(t)$ be the potential and energy associated with problem (1.1), respectively:

$$
\begin{gathered}
J(u)=\frac{1}{2}\left\|\nabla_{x} u\right\|_{2}^{2}+\frac{1}{2}\|\lambda(x) u\|_{2}^{2}-\frac{1}{p+1}\|u\|_{p+1}^{p+1} \quad \text { for } u \in H^{1}\left(\mathbb{R}^{n}\right), \\
E(t)=\frac{1}{2}\left\|u^{\prime}\right\|_{2}^{2}+J(u) .
\end{gathered}
$$

We get the local existence solution.

Theorem 2.5. Let $1<p \leq(n+2) /(n-2)(1<p<\infty$ if $n=1,2)$ and assume that $\left(u_{0}, u_{1}\right)$ $\in H^{1}\left(\mathbb{R}^{n}\right) \times L^{2}\left(\mathbb{R}^{n}\right)$ and $u_{0}$ belong to the modified stable set $\widetilde{W}^{*}$. Then there exists $T>0$ such that the Cauchy problem (1.1) has a unique solution $u(t)$ on $\mathbb{R}^{n} \times[0, T)$ in the class

$$
u(t, x) \in C\left([0, T) ; H^{1}\left(\mathbb{R}^{n}\right)\right) \cap C^{1}\left([0, T) ; L^{2}\left(\mathbb{R}^{n}\right)\right),
$$

satisfying

$$
u(t) \in \widetilde{\mathscr{W}}^{*},
$$

and this solution can be continued in time as long as $u(t) \in \widetilde{\mathscr{W}}^{*}$.

When $\lambda \neq$ const, we use the following theorem of local existence in the space $H^{2} \times H^{1}$, and the decay property of the energy $E(t)$ is necessarily required for the local solution to remain in $\widetilde{W}^{* *}$ as $t \rightarrow \infty$; this fact of course guarantees the global existence in $H^{2} \times H^{1}$ and by approximation, we obtain global existence in $H^{1} \times L^{1}$.

Theorem $2.6[15]$. Let $\left(u_{0}, u_{1}\right) \in H^{2} \times H^{1}$. Suppose that

$$
1 \leq p \leq \frac{n}{n-4} \quad(1 \leq \infty \text { if } N \leq 4) .
$$

Then under the hypotheses (2.1), (2.2), and (2.3), problem (1.1) admits a unique local solution $u(t)$ on some interval $\left[0, T\left[, T \equiv T\left(u_{0}, u_{1}\right)>0\right.\right.$, in the class $W^{2, \infty}\left(\left[0, T\left[; L^{2}\right) \cap W^{1, \infty}([0\right.\right.$, $T\left[; H^{1}\right) \cap L^{\infty}\left(\left[0, T\left[; H^{2}\right)\right.\right.$, satisfying the finite propagation property.

Proof of Theorem 2.5 (see $[15,18]$ ). Since the argument is standard, we only sketch the main idea of the proof. Let $\left(u_{0}, u_{1}\right) \in H^{1} \times L^{2}$ and $u_{0} \in \widetilde{W}^{*}$. Then we have a unique local solution $u(t)$ for some $T>0$. Indeed, taking suitable approximate functions $f_{j}$ such that (see $[20])$

$$
f_{j}(u)=f(u) \quad \text { if }|u| \leq j, \quad\left|f_{j}(u)\right| \leq|f(u)|, \quad\left|f_{j}(u)\right| \leq c_{j}|u|,
$$

problem $(1.1)$ with $f(u) \equiv|u|^{p-1} u$ replaced by $f_{j}(u)$ admits a unique solution $u_{j}(t) \in$ $C\left([0, T) ; H^{1}\left(\mathbb{R}^{n}\right)\right) \cap C^{1}\left([0, T) ; L^{2}\left(\mathbb{R}^{n}\right)\right)$. Further, we can prove that $u_{j}(t) \in \widetilde{W}^{*}, 0<t<T$, 
for sufficiently large $j$, and there exists a subsequence of $\left\{u_{j}(t)\right\}$ which converges to a function $\tilde{u}(t)$ in certain senses. $\tilde{u}(t)$ is, in fact, a weak solution in $C\left([0, T) ; H^{1}\left(\mathbb{R}^{n}\right)\right) \cap$ $C^{1}\left([0, T) ; L^{2}\left(\mathbb{R}^{n}\right)\right)($ see $[19,20])$ and such a solution is unique by Ginibre and Velo [5] and Brenner [3]. We can also construct such a solution which meets moreover the finite propagation property, if we assume that the initial data $u_{0}(x)$ and $u_{1}(x)$ are of compact support:

$$
\operatorname{supp} u_{0} \cup \operatorname{supp} u_{1} \subset\left\{x \in \mathbb{R}^{n},|x|<L\right\}, \quad \text { for some } L>0 \text {. }
$$

Applying [9, Appendix 1] of John, then the solution is also of compact support: supp $u_{j}(t)$ $\subset\left\{x \in \mathbb{R}^{n},|x|<L+t\right\}$. So, we have supp $\tilde{u}(t) \subset\left\{x \in \mathbb{R}^{n},|x|<L+t\right\}$.

We denote the life span of the solution $u(t, x)$ of the Cauchy problem (1.1) by $T_{\max }$. First we consider the case $\lambda(x) \equiv$ const $(\lambda(x) \equiv 1$ without loss of generality). And construct a stable set in $H^{1}\left(\mathbb{R}^{n}\right)$.

Setting

$$
\begin{gathered}
C_{0} \equiv K\left\{\frac{2(p+1)}{(p-1)}\right\}^{(p-1) / 2}, \\
\int_{0}^{\infty} \sigma(\tau) d \tau=+\infty \quad \text { if } m=1, \\
\int_{0}^{\infty}(1+\tau)^{-n(m-1) / 2} \sigma(\tau) d \tau=+\infty \quad \text { if } m>1 .
\end{gathered}
$$

Theorem 2.7. Let $u(t, x)$ be a local solution of problem (1.1) on $\left[0, T_{\max }\right)$ with initial data $u_{0} \in \widetilde{W}^{*}, u_{1} \in L^{2}\left(\mathbb{R}^{n}\right)$ with sufficiently small initial energy $E(0)$ so that

$$
C_{0} E(0)^{(p-1) / 2}<1
$$

Then $T_{\max }=\infty$. Furthermore, the global solution of the Cauchy problem (1.1) has the following energy decay property. Under (2.22), (2.3), and (2.23),

$$
E(t) \leq E(0) \exp \left(1-\omega \int_{0}^{t} \sigma(\tau) d \tau\right) \quad \forall t>0
$$

Under (2.2), (2.3), and (2.24),

$$
E(t) \leq\left(\frac{C(E(0))}{\int_{0}^{t}(1+\tau)^{-n(m-1) / 2} \sigma(\tau) d \tau}\right)^{2 /(m-1)} \quad \forall t>0 .
$$

Secondly, we consider the case $\lambda(x) \not \equiv$ const and we assume that

$$
\frac{n+4}{n} \leq p \leq \frac{n}{n-2} .
$$

(1) If $\sigma(t)=\mathcal{O}(\tilde{d}(t))$, where $\tilde{d}(t)=d(L+t)$. 
940 Cauchy problem for nonlinear wave equation

If $m=1$, we suppose that

$$
\int_{0}^{\infty} \sigma(\tau) d \tau=+\infty
$$

with

$$
\begin{gathered}
(\tilde{d}(t))^{-(4-(n-2)(p-1)) / 2} \exp \left(1-\omega \int_{0}^{t} \sigma(\tau) d \tau\right)^{(p-1) / 2}<\infty, \\
(\tilde{d}(t))^{-1} \exp \left(\frac{1}{2}-\frac{\omega}{2} \int_{0}^{t} \sigma(\tau) d \tau\right)<\infty
\end{gathered}
$$

If $m>1$, we suppose that

$$
\int_{0}^{\infty}(1+\tau)^{-n(m-1) / 2} \sigma(\tau) d \tau=\infty
$$

with

$$
\begin{gathered}
\frac{(\tilde{d}(t))^{-(4-(n-2)(p-1)) / 2}}{\left(\int_{0}^{t}(1+\tau)^{-n(m-1) / 2} \sigma(\tau) d \tau\right)^{(p-1) /(m-1)}}<\infty, \\
\frac{(\tilde{d}(t))^{-1}}{\left(\int_{0}^{t}(1+\tau)^{-n(m-1) / 2} \sigma(\tau) d \tau\right)^{1 /(m-1)}}<\infty .
\end{gathered}
$$

(2) If $\tilde{d}(t)=\sigma(\sigma(t))$.

If $m=1$, we suppose that for some $0 \leq \alpha<1$,

$$
\int_{0}^{\infty} \frac{\tilde{d}^{2}(\tau)}{\sigma^{\alpha}(\tau)} d \tau=+\infty
$$

with

$$
\begin{gathered}
(\tilde{d}(t))^{-(4-(n-2)(p-1)) / 2} \exp \left(1-\omega \int_{0}^{t} \frac{\tilde{d}^{2}(\tau)}{\sigma^{\alpha}(\tau)} d \tau\right)^{(p-1) / 2}<\infty, \\
(\tilde{d}(t))^{-1} \exp \left(\frac{1}{2}-\frac{\omega}{2} \int_{0}^{t} \frac{\tilde{d}^{2}(\tau)}{\sigma^{\alpha}(\tau)} d \tau\right)<\infty .
\end{gathered}
$$

If $m>1$, we suppose that for some $0 \leq \alpha<1$,

$$
\int_{0}^{\infty}(1+\tau)^{-n(m-1) / 2} \sigma^{-((1+\alpha)(1+m)-2) / 2}(\tau) \tilde{d}^{m+1}(\tau) d \tau=\infty
$$


with

$$
\begin{aligned}
& \frac{(\tilde{d}(t))^{-(4-(n-2)(p-1)) / 2}}{\left(\int_{0}^{t}(1+\tau)^{-n(m-1) / 2} \sigma^{-((1+\alpha)(1+m)-2) / 2}(\tau) \tilde{d}^{m+1}(\tau) d \tau\right)^{(p-1) /(m-1)}}<\infty, \\
& \frac{(\tilde{d}(t))^{-1}}{\left(\int_{0}^{t}(1+\tau)^{-n(m-1) / 2} \sigma^{-((1+\alpha)(1+m)-2) / 2}(\tau) \tilde{d}^{m+1}(\tau) d \tau\right)^{1 /(m-1)}}<\infty .
\end{aligned}
$$

We have the following theorem.

Theorem 2.8. Let $\left(u_{0}, u_{1}\right) \in H^{1} \times L^{2}, u_{0} \in \widetilde{W}^{* *}$, and let the initial energy $E(0)$ be sufficiently small. The following cases are considered.

(i) $\sigma(t)=\mathcal{O}(\tilde{d}(t))$.

Suppose (2.2), (2.3), (2.29), and (2.30) or (2.2), (2.3), (2.31), and (2.32). Then problem (1.1) admits a unique solution $u(t) \in C\left([0, \infty) ; H^{1}\right) \cap C^{1}\left([0, \infty) ; L^{2}\right)$ and has the same decay property as Theorem 2.7.

(ii) $\tilde{d}(t)=\tilde{O}(\sigma(t))$.

Suppose (2.2), (2.3), (2.33), and (2.34) or (2.2), (2.3), (2.35), and (2.36). Then problem (1.1) admits a unique solution $u(t) \in C\left([0, \infty) ; H^{1}\right) \cap C^{1}\left([0, \infty) ; L^{2}\right)$. Furthermore, the global solution of the Cauchy problem (1.1) has the following energy decay property:

$$
\begin{aligned}
& E(t) \leq E(0) \exp \left(1-\omega \int_{0}^{t} \frac{\tilde{d}^{2}(\tau)}{\sigma^{\alpha}(\tau)} d \tau\right) \quad \forall t>0 \text { if } m=1, \\
& E(t) \leq\left(\frac{C(E(0))}{\int_{0}^{t}(1+\tau)^{-n(m-1) / 2} \sigma^{-((1+\alpha)(1+m)-2) / 2}(\tau) \tilde{d}^{m+1}(\tau) d \tau}\right)^{2 /(m-1)} \quad \forall t>0 \text { if } m>1 .
\end{aligned}
$$

Remark 2.9. In Theorem 2.7, the global existence and energy decay are independent, but in Theorem 2.8, we need the estimation of the energy decay for a local solution to prove global existence.

Examples 2.10. (1) If $\sigma(t)=1 / t^{\theta}$, by applying Theorem 2.7 we obtain

$$
\begin{aligned}
& E(t) \leq E(0) e^{1-\omega t^{1-\theta}} \quad \text { if } m=1, \\
& E(t) \leq C(E(0))(1+t)^{-(2-n(m-1)-2 \theta) /(m-1)} \quad \text { if } 1<m<1+\frac{2-2 \theta}{n}, 0<\theta<1, \\
& E(t) \leq C(E(0))(\ln t)^{-2 /(m-1)} \quad \text { if } m=1+\frac{2-2 \theta}{n}, 0<\theta<1 .
\end{aligned}
$$

(2) If $\sigma(t)=1 / t^{\theta} \ln t \ln _{2} t \cdots \ln _{p} t$, by applying Theorem 2.7 , we obtain

$$
E(t) \leq E(0)\left(\ln _{p} t\right)^{-\omega} \quad \text { if } m=1, \theta=1 .
$$

For example, if $n(m-1) / 2+\theta=1$, that is, $1<m<1+2 / n$,

$$
E(t) \leq C(E(0))\left(\ln _{p} t\right)^{-2 /(m-1)}
$$


942 Cauchy problem for nonlinear wave equation

(3) If $\sigma(t)=1 / t^{\theta}$ and $d(r)=1 / r^{\gamma}$ with $\theta \geq \gamma$ by applying Theorem 2.8, we obtain

$$
\begin{gathered}
E(t) \leq C(E(0))(1+t)^{-(2-n(m-1)-2 \theta) /(m-1)} \quad \text { if } 1<m<1+\frac{2-2 \theta}{2 \gamma+n}, 0<\theta<1, \\
E(t) \leq C(E(0))(\ln t)^{-2 /(m-1)} \quad \text { if } m=1+\frac{2-2 \theta}{2 \gamma+n}, 0<\theta<1 .
\end{gathered}
$$

In order to show the global existence, it suffices to obtain the a priori estimates for $E(t)$ and $\|u(t)\|_{2}$ in the interval of existence.

To prove Theorem 2.7 we first have the following energy identity to problem (1.1).

Lemma 2.11 (energy identity). Let $u(t, x)$ be a local solution to problem (1.1) on [0, $\left.T_{\max }\right)$ as in Theorem 2.5. Then

$$
E(t)+\int_{\mathbb{R}^{n}} \int_{0}^{t} \sigma(s) u^{\prime}(s) g\left(u^{\prime}(s)\right) d s d x=E(0)
$$

for all $t \in\left[0, T_{\max }\right)$.

Next we state several facts about the modified stable set $\widetilde{W}^{*}$.

Lemma 2.12. Suppose that

$$
1<p \leq \frac{n+2}{n-2}
$$

Then

(i) $\widetilde{W}^{*}$ is a neighborhood of 0 in $H^{1}\left(\mathbb{R}^{n}\right)$,

(ii) for $u \in \widetilde{W}^{*}$,

$$
J(u) \geq \frac{p-1}{2(p+1)}\left(\left\|\nabla_{x} u\right\|_{2}^{2}+\|u\|_{2}^{2}\right) .
$$

Proof of Lemma 2.12. (i) From Lemma 2.1 we have

$$
\|u\|_{p+1}^{p+1} \leq K\|u\|_{H^{1}}^{p+1} \leq K\|u\|_{H^{1}}^{p-1}\left(\|u\|_{2}^{2}+\left\|\nabla_{x} u\right\|_{2}^{2}\right) .
$$

Let

$$
U(0) \equiv\left\{u \in H^{1}\left(\mathbb{R}^{N}\right) \backslash\|u\|_{H^{1}}^{p-1}<\frac{1}{K}\right\} .
$$

Then, for any $u \in U(0) \backslash\{0\}$, we deduce from (2.46) that

$$
\|u\|_{p+1}^{p+1}<\|u\|_{2}^{2}+\left\|\nabla_{x} u\right\|_{2}^{2}
$$

that is, $K(u)>0$. This implies $U(0) \subset \widetilde{\mathscr{W}}^{*}$.

(ii) By the definition of $K(u)$ and $J(u)$, we have the identity

$$
(p+1) J(u)=K(u)+\frac{(p-1)}{2}\left(\left\|\nabla_{x} u\right\|_{2}^{2}+\|u\|_{2}^{2}\right) .
$$


Since $u \in \widetilde{\mathscr{W}^{*}}$, we have $K(u) \geq 0$. Therefore from (2.44) we get the desired in-equality (2.45).

Lemma 2.13. Let $u(t)$ be a solution to problem (1.1) on [0, $\left.T_{\max }\right)$. Suppose (2.44) holds. If $u_{0} \in \widetilde{W}^{*}$ and $u_{1} \in L^{2}\left(\mathbb{R}^{n}\right)$ satisfy

$$
C_{0} E(0)^{(p-1) / 2}<1
$$

then

(i) $u(t) \in \widetilde{\mathscr{W}}^{*}$ on $\left[0, T_{\max }\right)$,

(ii) $\|u(t)\|_{2} \leq I_{0}$ on $\left[0, T_{\max }\right)$

Proof of Lemma 2.13. Suppose that there exists a number $t^{*} \in\left[0, T_{\max }[\right.$ such that $u(t) \in$ $\widetilde{W}^{*}$ on $\left[0, t^{*}\left[\right.\right.$ and $u\left(t^{*}\right) \notin \widetilde{W^{*}}$. Then we have

$$
K\left(u\left(t^{*}\right)\right)=0, \quad u\left(t^{*}\right) \neq 0 .
$$

Since $u(t) \in \widetilde{\mathscr{W}^{*}}$ on $\left[0, t^{*}[\right.$, it holds that

$$
\frac{p-1}{2(p+1)}\left(\left\|\nabla_{x} u\right\|_{2}^{2}+\|u\|_{2}^{2}\right) \leq J(u) \leq E(t)
$$

it follows from the nonincreasing of the energy that

$$
\left\|\nabla_{x} u\right\|_{2}^{2}+\|u\|_{2}^{2} \leq \frac{2(p+1)}{p-1} E(0) \equiv I_{0}^{2} .
$$

Hence, we obtain

$$
\|u\|_{2}^{2} \leq \frac{2(p+1)}{p-1} E(0) \equiv I_{0}^{2} \quad \text { on }\left[0, t^{*}\right] .
$$

Next, from Lemma 2.1 and (2.54) we have

$$
\begin{aligned}
\|u\|_{p+1}^{p+1} & \leq K\|u(t)\|_{H^{1}\left(\mathbb{R}^{n}\right)}^{p+1} \\
& \leq K\|u(t)\|_{H^{1}\left(\mathbb{R}^{n}\right)}^{p-1}\left(\left\|\nabla_{x} u\right\|_{2}^{2}+\|u\|_{2}^{2}\right) \\
& \leq K I_{0}^{p-1}\left(\left\|\nabla_{x} u\right\|_{2}^{2}+\|u\|_{2}^{2}\right) \\
& \leq C_{0} E(0)^{(p-1) / 2}\left(\|u(t)\|_{2}^{2}+\left\|\nabla_{x} u(t)\right\|_{2}^{2}\right)
\end{aligned}
$$

for all $t \in\left[0, t^{*}\right]$, where $C_{0}$ is the constant defined by (2.22). Note that from (2.55) and our hypothesis

$$
\eta_{0} \equiv C_{0} E(0)^{(p-1) / 2}<1
$$

it follows that

$$
\|u(t)\|_{p+1}^{p+1} \leq\left(1-\eta_{0}\right)\left(\|u(t)\|_{2}^{2}+\left\|\nabla_{x} u(t)\right\|_{2}^{2}\right)
$$


944 Cauchy problem for nonlinear wave equation

Therefore, we obtain

$$
K\left(u\left(t^{*}\right)\right) \geq \eta_{0}\left(\left\|u\left(t^{*}\right)\right\|_{2}^{2}+\left\|\nabla_{x} u\left(t^{*}\right)\right\|_{2}^{2}\right)
$$

which contradicts $(2.51)$. Thus, we conclude that $u(t) \in \widetilde{\mathscr{W}}^{*}$ on $\left[0, T_{\max }[\right.$. The assertion (ii) can be obtained by the same argument as for (2.54). This completes the proof of Lemma 2.13.

LEMmA 2.14. Under the same assumptions as in Lemma 2.13, there exists a constant $M_{2}$ depending on $\left\|u_{0}\right\|_{H^{1}}$ and $\left\|u_{1}\right\|_{2}$ such that

$$
\|u(t)\|_{H^{1}}^{2}+\left\|u^{\prime}(t)\right\|_{2}^{2} \leq M_{2}^{2}
$$

for all $t \in\left[0, T_{\max }[\right.$.

Proof of Lemma 2.14. It follows from Lemma 2.13 that $u(t) \in \widetilde{\mathscr{W}}^{*}$ on $\left[0, T_{\max }\right.$ [. So Lemma 2.12(ii) implies that

$$
J(u) \geq \frac{p-1}{2(p+1)}\left(\|u(t)\|_{2}^{2}+\left\|\nabla_{x} u(t)\right\|_{2}^{2}\right) \quad \text { on }\left[0, T_{\max }[\right.
$$

Hence, from Lemma 2.11 and (2.60) we get

$$
\frac{1}{2}\left\|u^{\prime}(t)\right\|_{2}^{2}+\frac{p-1}{2(p+1)}\left(\|u\|_{2}^{2}+\left\|\nabla_{x} u(t)\right\|_{2}^{2}\right) \leq E(t) \leq E(0) .
$$

So we get

$$
\|u(t)\|_{H^{1}}^{2}+\left\|u^{\prime}(t)\right\|_{2}^{2} \leq M_{2}^{2}
$$

for some $M_{2}>0$.

The above inequality and the continuation principle lead to the global existence of the solution, that is, $T_{\max }=\infty$.

Proof of the energy decay. From now on, we denote by $c$ various positive constants which may be different at different occurrences. We multiply the first equation of (1.1) by $E^{q} \phi^{\prime} u$, where $\phi$ is a function satisfying all the hypotheses of Lemma 2.4. We obtain

$$
\begin{aligned}
0= & \int_{S}^{T} E^{q} \phi^{\prime} \int_{\mathbb{R}^{n}} u\left(u^{\prime \prime}-\Delta u+u+\sigma(t) g\left(u^{\prime}\right)-|u|^{p-1} u\right) d x d t \\
= & {\left[E^{q} \phi^{\prime} \int_{\mathbb{R}^{n}} u u^{\prime} d x\right]_{S}^{T}-\int_{S}^{T}\left(q E^{\prime} E^{q-1} \phi^{\prime}+E^{q} \phi^{\prime \prime}\right) \int_{\mathbb{R}^{n}} u u^{\prime} d x d t-2 \int_{S}^{T} E^{q} \phi^{\prime} \int_{\mathbb{R}^{n}} u^{\prime 2} d x d t } \\
& +\int_{S}^{T} E^{q} \phi^{\prime} \int_{\mathbb{R}^{n}}\left(u^{\prime 2}+|u|^{2}+|\nabla u|^{2}-\frac{2}{p+1}|u|^{p+1}\right) d x d t+\int_{S}^{T} E^{q} \phi^{\prime} \int_{\mathbb{R}^{n}} \sigma(t) u g\left(u^{\prime}\right) d x d t \\
& \int_{S}^{T} E^{q} \phi^{\prime} \int_{\mathbb{R}^{n}}\left(\frac{2}{p+1}-1\right)|u|^{p+1} d x d t .
\end{aligned}
$$


Since

$$
\begin{aligned}
\left(1-\frac{2}{p+1}\right) \int_{\mathbb{R}^{n}}|u|^{p+1} d x & \leq\left(1-\eta_{0}\right) \frac{p-1}{p+1}\|u(t)\|_{H^{1}\left(\mathbb{R}^{n}\right)}^{2} d x \\
& \leq\left(1-\eta_{0}\right) \frac{p-1}{p+1} \frac{2(p+1)}{p-1} E(t) \\
& =2\left(1-\eta_{0}\right) E(t),
\end{aligned}
$$

we deduce that

$$
\begin{aligned}
2 \eta_{0} \int_{S}^{T} E^{q+1} \phi^{\prime} d t \leq & -\left[E^{q} \phi^{\prime} \int_{\mathbb{R}^{n}} u u^{\prime} d x\right]_{S}^{T}+\int_{S}^{T}\left(q E^{\prime} E^{q-1} \phi^{\prime}+E^{q} \phi^{\prime \prime}\right) \int_{\mathbb{R}^{n}} u u^{\prime} d x d t \\
& +2 \int_{S}^{T} E^{q} \phi^{\prime} \int_{\mathbb{R}^{n}} u^{\prime 2} d x d t-\int_{S}^{T} E^{q} \phi^{\prime} \int_{\mathbb{R}^{n}} \sigma(t) u g\left(u^{\prime}\right) d x d t \\
\leq & -\left[E^{q} \phi^{\prime} \int_{\mathbb{R}^{n}} u u^{\prime} d x\right]_{S}^{T}+\int_{S}^{T}\left(q E^{\prime} E^{q-1} \phi^{\prime}+E^{q} \phi^{\prime \prime}\right) \int_{\mathbb{R}^{n}} u u^{\prime} d x d t \\
& +2 \int_{S}^{T} E^{q} \phi^{\prime} \int_{\mathbb{R}^{n}} u^{\prime 2} d x d t+c(\varepsilon) \int_{S}^{T} E^{q} \phi^{\prime} \int_{\left|u^{\prime}\right| \leq 1} g\left(u^{\prime}\right)^{2} d x d t \\
& +\varepsilon \int_{S}^{T} E^{q} \phi^{\prime} \int_{\mathbb{R}^{n}} u^{2} d x d t+\int_{S}^{T} E^{q} \phi^{\prime} \int_{\left|u^{\prime}\right| \geq 1} \sigma(t) u g\left(u^{\prime}\right) d x d t
\end{aligned}
$$

for every $\varepsilon>0$. Also, applying Hölder's and Young's inequalities, we have

$$
\begin{aligned}
\int_{S}^{T} E^{q} & \phi^{\prime} \int_{\left|u^{\prime}\right|>1} \sigma(t) u g\left(u^{\prime}\right) d x d t \\
& \leq \int_{S}^{T} E^{q} \phi^{\prime} \sigma(t)\left(\int_{\Omega}|u|^{r+1} d x\right)^{1 /(r+1)}\left(\int_{\left|u^{\prime}\right|>1}\left|g\left(u^{\prime}\right)\right|^{(r+1) / r} d x\right)^{r /(r+1)} d t \\
& \leq c \int_{S}^{T} E^{(2 q+1) / 2} \phi^{\prime} \sigma^{1 /(r+1)}(t)\left(\int_{\left|u^{\prime}\right|>1} \sigma(t) u^{\prime} g\left(u^{\prime}\right) d x\right)^{r /(r+1)} d t \\
& \leq \int_{S}^{T} \phi^{\prime} \sigma^{1 /(r+1)}(t) E^{(2 q+1) / 2}\left(-E^{\prime}\right)^{r /(r+1)} d t \\
& \leq c \int_{S}^{T} \phi^{\prime}\left(\sigma^{1 /(r+1)}(t) E^{(2 q+1) / 2-r /(r+1)}\right)\left(\left(-E^{\prime}\right)^{r /(r+1)} E^{r /(r+1)}\right) d t \\
& \leq c\left(\varepsilon^{\prime}\right) \int_{S}^{T} \phi^{\prime}\left(-E^{\prime} E\right) d t+\varepsilon^{\prime} \int_{S}^{T} \phi^{\prime} \sigma(t) E^{(r+1)((2 q+1) / 2-r /(r+1))} d t \\
& \leq c\left(\varepsilon^{\prime}\right) E(S)^{2}+\varepsilon^{\prime} \sigma(0) E(0)^{(2 r q-r-1) / 2} \int_{S}^{T} \phi^{\prime} E^{q+1} d t
\end{aligned}
$$


946 Cauchy problem for nonlinear wave equation

for every $\varepsilon^{\prime}>0$. Choosing $\varepsilon$ and $\varepsilon^{\prime}$ small enough, we obtain

$$
\begin{aligned}
\int_{S}^{T} E^{q+1} \phi^{\prime} d t \leq & -\left[E^{q} \phi^{\prime} \int_{\mathbb{R}^{n}} u u^{\prime} d x\right]_{S}^{T}+\int_{S}^{T}\left(q E^{\prime} E^{q-1} \phi^{\prime}+E^{q} \phi^{\prime \prime}\right) \int_{\mathbb{R}^{n}} u u^{\prime} d x d t \\
& +\int_{\left|u^{\prime}\right| \geq 1} \sigma(t) u g\left(u^{\prime}\right) d x d t+c \int_{S}^{T} E \phi^{\prime} \int_{\mathbb{R}^{n}} u^{\prime 2} d x d t \\
\leq & c E(S)+c \int_{S}^{T} E \phi^{\prime} \int_{\mathbb{R}^{n}} u^{\prime 2} d x d t .
\end{aligned}
$$

Since $x g(x) \geq 0$ for all $x \in \mathbb{R}$, it follows that the energy is nonincreasing, locally absolutely continuous and $E^{\prime}(t)=-\int_{\mathbb{R}^{n}} \sigma(t) u^{\prime} g\left(u^{\prime}\right) d x$ a.c. in $\mathbb{R}_{+}$.

Proof of (2.26). We consider the case $m=1$, that is,

$$
c_{3}|v| \leq|g(v)| \leq c_{4}|v| \quad \text { for all }|v| \leq 1
$$

Then we have

$$
u^{\prime 2} \leq \frac{c_{13}}{\sigma(t)} u^{\prime} \rho\left(t, u^{\prime}\right) \quad \forall t \in \mathbb{R}, \forall x \in \mathbb{R}^{n}
$$

where $\rho(t, s)=\sigma(t) g(s)$ for all $s \in \mathbb{R}$. Therefore we deduce from (2.67) (applied with $q=0$ ) that

$$
\int_{S}^{T} E(t) \phi^{\prime}(t) d t \leq C E(S)+2 C \int_{S}^{T} \phi^{\prime}(t) \int_{\mathbb{R}^{n}} \frac{1}{\sigma(t)} u^{\prime} \rho\left(t, u^{\prime}\right) d x d t
$$

Define

$$
\phi(t)=\int_{0}^{t} \sigma(\tau) d \tau
$$

It is clear that $\phi$ is a nondecreasing function of class $C^{2}$ on $\mathbb{R}_{+}$. The hypothesis $(2.23)$ ensures that

$$
\phi(t) \longrightarrow+\infty \quad \text { as } t \longrightarrow+\infty
$$

Then we deduce from (2.70) that

$$
\int_{S}^{T} E(t) \phi^{\prime}(t) d t \leq C E(S)+2 C \int_{S}^{T} \int_{\mathbb{R}^{n}} u^{\prime} \rho\left(t, u^{\prime}\right) d x d t \leq 3 C E(S),
$$

and thanks to Lemma 2.4 we obtain

$$
E(t) \leq E(0) e^{(1-\phi(t)) /(3 C)}
$$

Proof of (2.27). Now we assume that $m>1$ in (2.2). Define $\phi$ by (2.71). We apply Lemma 2.4 with $q=(m-1) / 2$. 
We need to estimate

$$
\int_{S}^{T} E^{q} \phi^{\prime} \int_{\mathbb{R}^{n}} u^{\prime 2} d x d t
$$

For $t \geq 0$, consider

$$
\Omega_{1}=\left\{x \in \mathbb{R}^{n},\left|u^{\prime}\right| \leq 1\right\}, \quad \Omega_{2}=\left\{x \in \mathbb{R}^{n},\left|u^{\prime}\right|>1\right\} .
$$

First we note that for every $t \geq 0$,

$$
\Omega_{1} \cup \Omega_{2}=\mathbb{R}^{n} .
$$

Next we deduce from (2.2) and (2.3) that for every $t \geq 0$,

(i) if $x \in \Omega_{1}$, then $u^{\prime 2} \leq\left((1 / \sigma(t)) u^{\prime} \rho\left(t, u^{\prime}\right)\right)^{2 /(m+1)}$,

(ii) if $x \in \Omega_{2}$, then $u^{\prime 2} \leq(1 / \sigma(t)) u^{\prime} \rho\left(t, u^{\prime}\right)$.

Hence, using Hölder's inequality, we get that

$$
\begin{aligned}
\int_{S}^{T} E^{q} \phi^{\prime} & \int_{\mathbb{R}^{n}} u^{\prime 2} d x d t \\
\leq & 2 \int_{S}^{T} E^{q} \phi^{\prime} \int_{\mathbb{R}^{n}} \frac{1}{\sigma(t)} u^{\prime} \rho\left(t, u^{\prime}\right) d x d t+2 \int_{S}^{T} E^{q} \phi^{\prime} \int_{\mathbb{R}^{n}}\left(\frac{1}{\sigma(t)} u^{\prime} \rho\left(t, u^{\prime}\right)\right)^{2 /(m+1)} d x d t \\
\leq & 2 \int_{S}^{T} E^{q} \phi^{\prime} \int_{\mathbb{R}^{n}} \frac{1}{\sigma(t)} u^{\prime} \rho\left(t, u^{\prime}\right) d x d t+2 \int_{S}^{T} E^{q} \phi^{\prime} \int_{\{|x| \leq(L+t)\}}\left(u^{\prime} g\left(u^{\prime}\right)\right)^{2 /(m+1)} d x d t \\
\leq & 2 \int_{S}^{T} E^{q} \phi^{\prime} \int_{\mathbb{R}^{n}} \frac{1}{\sigma(t)} u^{\prime} \rho\left(t, u^{\prime}\right) d x d t \\
& +2 \int_{S}^{T} E^{q} \phi^{\prime}(1+t)^{n(m-1) /(m+1)}\left(\int_{\mathbb{R}^{n}} u^{\prime} g\left(u^{\prime}\right) d x\right)^{2 /(m+1)} d t \\
\leq & c E(S)^{1+q}+c^{\prime} \int_{S}^{T} E^{q} \phi^{\prime}(1+t)^{n(m-1) /(m+1)}\left(\frac{-E^{\prime}}{\sigma(t)}\right)^{2 /(m+1)} d t \\
\leq & c E(S)^{1+q}+c^{\prime} \int_{S}^{T} E^{q} \phi^{\prime}(1+t)^{n(m-1) /(m+1)} \sigma^{-2 /(m+1)}(t)\left(-E^{\prime}\right)^{2 /(m+1)} d t .
\end{aligned}
$$

Set $\varepsilon>0$; thanks to Young's inequality and to our definitions of $p$ and $\phi$, we obtain

$$
\begin{aligned}
\int_{S}^{T} E^{q} \phi^{\prime} & \int_{\mathbb{R}^{n}} u^{\prime 2} d x d t \\
\leq & c E(S)^{1+q}+2 \frac{m-1}{m+1} \varepsilon^{(m+1) /(m-1)} \int_{S}^{T} E^{1+q}\left(\phi^{\prime}\right)^{(m+1) /(m-1)}(1+t)^{n} \sigma^{-2 /(m-1)} d t \\
& +\frac{4}{m+1} \frac{1}{\varepsilon^{(m+1) / 2}} E(S) .
\end{aligned}
$$

We choose $\phi^{\prime}$ such that

$$
\phi^{\prime 2 /(m-1)}(1+t)^{n} \sigma^{-2 /(m-1)}=1,
$$


so

$$
\phi(t)=\int_{0}^{t}(1+s)^{-n(m-1) / 2} \sigma(s) d s .
$$

Then we deduce from (2.79) that

$$
\int_{S}^{T} E^{1+q} \phi^{\prime} d t \leq 2 C E(S),
$$

and thanks to Lemma 2.4 (applied with $c=0$ ) we obtain

$$
E(t) \leq \frac{C}{\phi(t)^{2 /(m-1)}}
$$

Proof of Theorem 2.8. First, we see that if $u \in \widetilde{\mathscr{W}}^{* *}$, then

$$
\left\|\nabla_{x} u\right\|_{2}^{2}+2 \int_{\mathbb{R}^{n}} F(u(t)) d x \geq\left\|\nabla_{x} u\right\|_{2}^{2}-\frac{2}{p+1}\|u(t)\|_{p+1}^{p+1} \geq \frac{p-1}{p+1}\left\|\nabla_{x} u\right\|_{2}^{2} .
$$

In the proof, we often use the following inequality:

$$
\|u(t)\|_{2} \leq \frac{1}{\tilde{d}(t)}\|\lambda(x) u(t)\|_{2}
$$

Now, we assume that $I\left(u_{0}\right)>(1 / 2)\left\|\nabla_{x} u_{0}\right\|_{2}^{2}$. Then

$$
I(u(t)) \geq \frac{1}{2}\left\|\nabla_{x} u(t)\right\|_{2}^{2}
$$

for some interval near $t=0$. As long as (2.86) holds, we have $J(t) \equiv I(u(t))$. Thus

$$
\begin{aligned}
2 \eta_{0} \int_{S}^{T} E^{q+1} \phi^{\prime} d t \leq & -\left[E^{q} \phi^{\prime} \int_{\mathbb{R}^{n}} u u^{\prime} d x\right]_{S}^{T}+\int_{S}^{T}\left(q E^{\prime} E^{q-1} \phi^{\prime}+E^{q} \phi^{\prime \prime}\right) \int_{\mathbb{R}^{n}} u u^{\prime} d x d t \\
& +2 \int_{S}^{T} E^{q} \phi^{\prime} \int_{\mathbb{R}^{n}} u^{\prime 2} d x d t-\int_{S}^{T} E^{q} \phi^{\prime} \int_{\mathbb{R}^{n}} \sigma(t) u g\left(u^{\prime}\right) d x d t \\
\leq & -\left[E^{q} \phi^{\prime} \int_{\mathbb{R}^{n}} u u^{\prime} d x\right]_{S}^{T}+\int_{S}^{T}\left(q E^{\prime} E^{q-1} \phi^{\prime}+E^{q} \phi^{\prime \prime}\right) \int_{\mathbb{R}^{n}} u u^{\prime} d x d t \\
& +2 \int_{S}^{T} E^{q} \phi^{\prime} \int_{\mathbb{R}^{n}} u^{\prime 2} d x d t+c(\varepsilon) \int_{S}^{T} E^{q} \phi^{\prime} \int_{\left|u^{\prime}\right| \leq 1}\left(\frac{\sigma(t)}{\lambda(x)}\right)^{2} g\left(u^{\prime}\right)^{2} d x d t \\
& +\varepsilon \int_{S}^{T} E^{q} \phi^{\prime} \int_{\mathbb{R}^{n}} \lambda^{2}(x) u^{2} d x d t+\int_{S}^{T} E^{q} \phi^{\prime} \int_{\left|u^{\prime}\right| \geq 1} \sigma(t) u g\left(u^{\prime}\right) d x d t .
\end{aligned}
$$

If $\sigma(t)=\mathcal{O}(\tilde{d}(t))$, that is, $\sigma(t) \rightarrow 0$ as $t \rightarrow \infty$ more rapidly than $\tilde{d}(t)$, we find the same results of asymptotic behaviour as in Theorem 2.7. 
If $\tilde{d}(t)=\mathscr{O}(\sigma(t))$, so, we obtain

$$
\begin{aligned}
& \int_{S}^{T} E^{q+1} \phi^{\prime} d t \leq-\left[E^{q} \phi^{\prime} \int_{\mathbb{R}^{n}} u u^{\prime} d x\right]_{S}^{T}+\int_{S}^{T}\left(q E^{\prime} E^{q-1} \phi^{\prime}+E^{q} \phi^{\prime \prime}\right) \int_{\mathbb{R}^{n}} u u^{\prime} d x d t \\
& \int_{S}^{T} E^{q} \phi^{\prime} \int_{\left|u^{\prime}\right| \geq 1} u^{\prime 2} d x d t \int_{S}^{T} E^{q} \phi^{\prime} \int_{\left|u^{\prime}\right| \geq 1} \sigma(t) u g\left(u^{\prime}\right) d x d t \\
& \quad+c(\varepsilon) \int_{S}^{T} E^{q} \phi^{\prime} \int_{\left|u^{\prime}\right| \leq 1}\left(\frac{\sigma(t)}{\lambda(x)}\right)^{2}\left|u^{\prime}\right|^{2} d x d t .
\end{aligned}
$$

We consider the case $m=1$. Thus under (2.2) and (2.3), we have

$$
\int_{S}^{T} E^{q} \phi^{\prime} \int_{\mathbb{R}^{n}}\left(\frac{\sigma(t)}{\lambda(x)}\right)^{2} u^{\prime 2} d x d t \leq C \int_{S}^{T} E^{q} \phi^{\prime} \int_{\mathbb{R}^{n}}\left(\frac{\sigma^{\alpha}(t)}{\tilde{d}^{2}(t)}\right) \sigma(t) u^{\prime} g\left(u^{\prime}\right) d x d t
$$

for all $0 \leq \alpha<1$. We choose

$$
\phi(t)=\int_{0}^{t} \frac{\tilde{d}^{2}(s)}{\sigma^{\alpha}(s)} d s
$$

It is clear that $\phi$ is a nondecreasing function of class $C^{2}$ on $\mathbb{R}_{+}$. Hypothesis (2.33) ensures that

$$
\phi(t) \longrightarrow+\infty \quad \text { as } t \longrightarrow+\infty
$$

By (2.85), the definition of $E$, and the Cauchy-Schwartz inequality, we have

$$
\begin{aligned}
&-\left[E^{q} \phi^{\prime} \int_{\mathbb{R}^{n}} u u^{\prime} d x\right]_{S}^{T}=E^{q}(S) \phi^{\prime}(S) \int_{\mathbb{R}^{n}} u(S) u^{\prime}(S) d x-E^{q}(T) \phi^{\prime}(T) \int_{\mathbb{R}^{n}} u(T) u^{\prime}(T) d x \\
& \leq C E^{q+1}(S)\left[\frac{\phi^{\prime}(S)}{\tilde{d}(S)}+\frac{\phi^{\prime}(T)}{\tilde{d}(T)}\right] \leq C E^{q+1}(S), \\
& \int_{S}^{T}\left(q E^{\prime} E^{q-1} \phi^{\prime}+E^{q} \phi^{\prime \prime}\right) \int_{\mathbb{R}^{n}} u u^{\prime} d x d t \leq \int_{S}^{T} q\left|E^{\prime}\right| E^{q} \frac{\phi^{\prime}(t)}{\tilde{d}(t)} d t+\int_{S}^{T} E^{q+1} \frac{\left|\phi^{\prime \prime}(t)\right|}{\tilde{d}(t)} d t
\end{aligned}
$$

when we have (in the case $m=1$ )

$$
\phi^{\prime}=\frac{\tilde{d}^{2}(t)}{\sigma^{\alpha}(t)}, \quad \phi^{\prime \prime}(t)=\frac{2 \tilde{d}(t) \tilde{d}^{\prime}(t)}{\sigma^{\alpha}(t)}-\alpha \frac{\sigma^{\prime}(t) \tilde{d}^{2}(t)}{\sigma^{\alpha+1}(t)} .
$$

So

$$
\left|\frac{\phi^{\prime \prime}(t)}{\tilde{d}(t)}\right| \leq-\frac{2 \tilde{d}^{\prime}(t)}{\tilde{d}^{\alpha}(t)} \frac{\tilde{d}^{\alpha}(t)}{\sigma^{\alpha}(t)}-\alpha \frac{\tilde{d}(t)}{\sigma(t)} \frac{\sigma^{\prime}(t)}{\sigma^{\alpha}(t)},
$$


$\tilde{d}(t) / \sigma(t)$ is bounded, so we obtain

$$
\begin{aligned}
\int_{S}^{T} E^{q+1} \frac{\left|\phi^{\prime \prime}(t)\right|}{\tilde{d}(t)} d t & \leq-E^{q+1}(S)\left[\tilde{d}^{1-\alpha}(t)+\sigma^{1-\alpha}(t)\right]_{S}^{T} \\
& \leq E^{q+1}(S)\left(\tilde{d}^{1-\alpha}(t)+\sigma^{1-\alpha}(t)\right) \\
& \leq C E^{q+1}(S) .
\end{aligned}
$$

Then we deduce from (2.88) that

$$
\int_{S}^{T} E \phi^{\prime} d t \leq C E(S)+2 C \int_{S}^{T} \int_{\mathbb{R}^{n}} \sigma(t) u^{\prime} g\left(u^{\prime}\right) d x d t \leq 3 C E(S),
$$

and thanks to Lemma 2.4 we obtain

$$
E(t) \leq E(0) e^{(1-\phi(t)) / 3 C}
$$

Using the condition that $\tilde{d}(t)=\mathscr{O}(\sigma(t))$ and using Hölder's inequality, we get that

$$
\begin{aligned}
\int_{S}^{T} E^{q} & \phi^{\prime} \int_{\left|u^{\prime}\right| \leq 1} \frac{\sigma^{2}(t)}{\lambda^{2}(x)} u^{\prime 2} d x d t \\
& \leq 2 \int_{S}^{T} E^{q} \phi^{\prime} \int_{\mathbb{R}^{n}} \frac{\sigma^{2}(t)}{\lambda^{2}(x)}\left(\frac{1}{\sigma(t)} \sigma(t) u^{\prime} g\left(u^{\prime}\right)\right)^{2 /(m+1)} d x d t \\
& \leq 2 \int_{S}^{T} E^{q} \phi^{\prime} \int_{\{|x| \leq L+t\}} \frac{\sigma^{2}(t)}{\lambda^{2}(x)}\left(\frac{1}{\sigma(t)} \sigma(t) u^{\prime} g\left(u^{\prime}\right)\right)^{2 /(m+1)} d x d t \\
& \leq 2 \int_{S}^{T} E^{q} \phi^{\prime}\left(\frac{\sigma(t)}{\tilde{d}(t)}\right)^{2}(1+t)^{n(m-1) /(m+1)}\left(\int_{\mathbb{R}^{n}} u^{\prime} g\left(u^{\prime}\right) d x\right)^{2 /(m+1)} d t \\
& \leq c^{\prime} \int_{S}^{T} E^{q} \phi^{\prime}\left(\frac{\sigma(t)}{\tilde{d}(t)}\right)^{2}(1+t)^{n(m-1) /(m+1)}\left(\frac{-E^{\prime}}{\sigma(t)}\right)^{2 /(m+1)} d t \\
& \leq c^{\prime} \int_{S}^{T} E^{q} \phi^{\prime}\left(\frac{\sigma(t)}{\tilde{d}(t)}\right)^{2}(1+t)^{n(m-1) /(m+1)} \sigma^{-2 /(m+1)}(t)\left(-E^{\prime}\right)^{2 /(m+1)} d t \\
& \leq c^{\prime} \int_{S}^{T} E^{q} \phi^{\prime} \frac{\sigma^{\alpha+1}(t)}{\tilde{d}^{2}(t)}(1+t)^{n(m-1) /(m+1)} \sigma^{-2 /(m+1)}(t)\left(-E^{\prime}\right)^{2 /(m+1)} d t .
\end{aligned}
$$

Set $\varepsilon>0$; thanks to Young's inequality and to our definitions of $p$ and $\phi$, we obtain

$$
\begin{aligned}
\int_{S}^{T} & E^{q} \phi^{\prime} \int_{\mathbb{R}^{n}}\left(\frac{\sigma(t)}{\lambda(x)}\right)^{2} u^{\prime 2} d x d t \\
\leq & c E(S)^{1+q}+2 \frac{m-1}{m+1} \varepsilon^{(m+1) /(m-1)} \\
\quad & \times \int_{S}^{T} E^{1+q}\left(\phi^{\prime}\right)^{(m+1) /(m-1)} \frac{\sigma^{(1+\alpha)(m+1) /(m-1)}(t)}{\tilde{d}^{2(m+1) /(m-1)}(t)}(1+t)^{n} \sigma^{-2 /(m-1)} d t+\frac{4}{m+1} \frac{1}{\varepsilon^{(m+1) / 2}} E(S) .
\end{aligned}
$$


We choose $\phi$ such that

$$
\phi(t)=\int_{0}^{t}(1+\tau)^{-n(m-1) / 2} \sigma^{-((1+\alpha)(m+1)-2) / 2}(\tau) \tilde{d}^{m+1}(\tau) d \tau .
$$

It is clear that $\phi$ is a nondecreasing function of class $C^{2}$ on $\mathbb{R}_{+}$. The hypothesis (2.35) ensures that $\phi(t) \rightarrow+\infty$ as $t \rightarrow+\infty$. By (2.85), the definition of $E$, and the Cauchy-Schwartz inequality we have (2.92) when we have (the case $m>1$ )

$$
\begin{aligned}
\phi^{\prime}=(1+t)^{-n(m-1) / 2} \sigma^{-((1+\alpha)(m+1)-2) / 2}(t) \tilde{d}^{m+1}(t), & \\
\phi^{\prime \prime}(t)= & -\frac{n(m-1)}{2}(1+t)^{-n(m-1) / 2-1} \sigma^{-((1+\alpha)(m+1)-2) / 2}(t) \tilde{d}^{m+1}(t) \\
+(1+t)^{-n(m-1) / 2}( & -\frac{(1+\alpha)(m+1)-2}{2} \sigma^{-(1+\alpha)(m+1) / 2}(t) \sigma^{\prime}(t) \tilde{d}^{m+1}(t) \\
& \left.+(m+1) \tilde{d}^{m}(t) \tilde{d}^{\prime}(t) \sigma^{-((1+\alpha)(m+1)-2) / 2}(t)\right) .
\end{aligned}
$$

Thus

$$
\begin{aligned}
\left|\frac{\phi^{\prime \prime}(t)}{\tilde{d}(t)}\right| \leq & C \frac{\tilde{d}^{m}(t)}{\sigma^{m}(t)} \sigma^{(1-\alpha)(m+1) / 2}(t)-C^{\prime} \frac{\tilde{d}^{m}(t)}{\sigma^{m}(t)} \frac{\sigma^{\prime}(t)}{\sigma^{((1+\alpha)(m+1)-2 m) / 2}(t)} \\
& -C^{\prime \prime} \frac{\tilde{d}^{((1+\alpha)(m+1)-2) / 2}(t)}{\sigma^{((1+\alpha)(m+1)-2) / 2}(t)} \frac{\tilde{d}^{\prime}(t)}{\tilde{d}((1+\alpha)(m+1)-2 m) / 2(t)},
\end{aligned}
$$

$\tilde{d}(t) / \sigma(t)$ is bounded, so we obtain

$$
\begin{aligned}
\int_{S}^{T} E^{q+1} \frac{\left|\phi^{\prime \prime}(t)\right|}{\tilde{d}(t)} d t & \leq-E^{q+1}(S)\left[\tilde{d}^{(1-\alpha)(m+1) / 2}(t)+\sigma^{(1-\alpha)(m+1) / 2}(t)\right]_{S}^{T} \\
& \leq E^{q+1}(S)\left(\tilde{d}^{(1-\alpha)(m+1) / 2(S)+\sigma^{(1-\alpha)(m+1) / 2}(S)}\right) \\
& \leq C E^{q+1}(S) .
\end{aligned}
$$

We deduce from this choice

$$
\int_{S}^{T} E^{1+q} \phi^{\prime} d t \leq 2 C E(S)
$$

and thanks to Lemma 2.4 (applied with $c=0$ ), we obtain

$$
E(t) \leq \frac{C(E(0))}{\phi^{2 /(m-1)}} .
$$

Since $u_{0} \in \widetilde{\mathscr{W}}^{* *}$ and $\widetilde{\mathscr{W}^{*} *}$ is an open set, putting

$$
T_{1}=\sup \left\{t \in[0,+\infty): u(s) \in \widetilde{\mathscr{W}}^{* *} \text { for } 0 \leq s \leq t\right\},
$$

we see that $T_{1}>0$ and $u(t) \in \widetilde{\mathscr{W}}^{* *}$ for $0 \leq t<T_{1}$. If $T_{1}<T_{\max }<\infty$, where $T_{\max }$ is the lifespan of the solution, then $u\left(T_{1}\right) \in \widetilde{\mathscr{W}}^{* *}$; that is,

$$
I\left(u\left(T_{1}\right)\right)=0, \quad u\left(T_{1}\right) \neq 0 .
$$


We see from Lemma 2.2 and (2.85) that

$$
\begin{aligned}
\|u(t)\|_{p+1}^{p+1} & \leq C\|u(t)\|_{2}^{(4-(n-2)(p-1)) / 2}\left\|\nabla_{x} u(t)\right\|_{2}^{n(p-1) / 2} \\
& \leq(\tilde{d}(t))^{-(4-(n-2)(p-1)) / 2} E^{(p-1) / 2}\left\|\nabla_{x} u(t)\right\|_{2}^{2} \\
& \leq B(t)\left\|\nabla_{x} u(t)\right\|_{2}^{2}
\end{aligned}
$$

for $0 \leq t \leq T_{1}$, where we have used the assumption $p \geq(n+4) / n$ and

$$
B(t)=\frac{C(E(0)) \tilde{d}^{-(4-(n-2)(p-1)) / 2}(t)}{\left(\int_{0}^{t}(1+\tau)^{-n(m-1) / 2} \sigma^{-((1+\alpha)(m+1)-2) / 2}(\tau) \tilde{d}^{m+1}(\tau) d \tau\right)^{(p-1) /(m-1)}} .
$$

Next, we put

$$
T_{2} \equiv \sup \left\{t \in[0,+\infty): B(s)<\frac{1}{2} \text { for } 0 \leq s<t\right\},
$$

and then we see that $T_{2}>0$ and $T_{2}=T_{1}$ because $B(t)<1 / 2$ by the condition that $E(0)$ is small. Then

$$
I(u(t)) \geq\left\|\nabla_{x} u(t)\right\|_{2}^{2}-B(t)\left\|\nabla_{x} u(t)\right\|_{2}^{2} \geq \frac{1}{2}\left\|\nabla_{x} u(t)\right\|_{2}^{2}
$$

for $0 \leq t \leq T_{1}$. Moreover, (2.107) and (2.111) imply

$$
K\left(u\left(T_{1}\right)\right) \geq \frac{1}{2}\left\|\nabla_{x} u\left(T_{1}\right)\right\|_{2}^{2}>0,
$$

which is a contradiction, and hence, it might be $T_{1}=T_{\max }$. Therefore, (2.105) holds true for $0 \leq T \leq T_{\max }$. To prove global existence in $H^{2} \cap H^{1}$, we need to derive the estimates for second derivatives of $u(t)$ on the basis of the energy estimate of $E(t)$, we utilize the differentiated equation

$$
u_{t t t}-\Delta_{x} u^{\prime}+\lambda^{2}(x) u^{\prime}+\sigma^{\prime}(t) g\left(u^{\prime}\right)+\sigma(t) g\left(u^{\prime}\right) u^{\prime \prime}+f^{\prime}(u) u^{\prime}=0,
$$

where $f(u)=|u|^{p-1} u$. Multiplying (2.113) by $u^{\prime \prime}$, we have

$$
\begin{aligned}
& \frac{d}{d t} E_{2}(t)+2 \sigma(t) \int_{\mathbb{R}^{n}} g^{\prime}\left(u^{\prime}\right)\left|u^{\prime \prime}(t)\right|^{2} d x \\
& \quad \leq 2 \int_{\mathbb{R}^{n}}\left|f^{\prime}(u)\right|\left|u^{\prime}(t)\right|\left|u^{\prime \prime}(t)\right| d x+2\left|\sigma^{\prime}(t)\right| \int_{\mathbb{R}^{n}}\left|g\left(u^{\prime}\right)\right|\left|u^{\prime \prime}(t)\right| d x,
\end{aligned}
$$

where we set

$$
E_{2}(t)=\left\|u^{\prime \prime}(t)\right\|_{2}^{2}+\left\|\nabla_{x} u(t)\right\|_{2}^{2}+\left\|\lambda u^{\prime}(t)\right\|_{2}^{2} .
$$

By (2.2) and (2.3), we have

$$
\begin{array}{r}
\int_{\mathbb{R}^{n}}\left|g\left(u^{\prime}\right)\right|^{2} d x \leq C \int_{\left|u^{\prime}\right| \leq 1}\left|u^{\prime}\right|^{2 / m} d x+C^{\prime} \int_{\left|u^{\prime}\right| \geq 1}\left|u^{\prime}\right|^{2 r} d x \\
\leq C(L+t)^{n(m-1) / m} E^{1 / m}+C^{\prime} E^{(2-(n-2)(r-1)) / 2} E_{2}^{n(r-1) / 2}
\end{array}
$$


and by Lemma 2.2

$$
\begin{aligned}
2 \int_{\mathbb{R}^{n}}\left|f^{\prime}(u)\right|\left|u^{\prime}(t)\right|\left|u^{\prime \prime}(t)\right| d x & \leq C\|u(t)\|_{n(p-1)}^{p-1}\left\|u^{\prime}(t)\right\|_{2 n /(n-2)}\left\|u^{\prime \prime}(t)\right\|_{2} \\
& \leq c\|u(t)\|_{n(p-1)}^{p-1}\left\|\nabla_{x} u^{\prime}(t)\right\|_{2}\left\|u^{\prime \prime}(t)\right\|_{2} \\
& \leq c\|u(t)\|_{n(p-1)}^{p-1} E_{2}(t) .
\end{aligned}
$$

Since $(n+2) / n \leq p \leq n /(n-2)$, then

$$
\|u(t)\|_{n(p-1)}^{p-1} \leq \tilde{d}^{-(2-(n-2)(p-1)) / 2} E^{(p-1) / 2} .
$$

Thus, we have

$$
\begin{aligned}
\frac{d}{d t} E_{2} \leq & \tilde{d}^{-(2-(n-2)(p-1)) / 2} E^{(p-1) / 2} E_{2}(t) \\
& +2\left|\sigma^{\prime}(t)\right|\left((L+t)^{n(m-1) / m} E^{1 / m}+C E^{(2-(n-2)(r-1)) / 2} E_{2}^{n(r-1) / 2}\right)^{1 / 2} E_{2}^{1 / 2}(t) .
\end{aligned}
$$

We have also, applying Young inequality,

$$
E^{(2-(n-2)(r-1)) / 2} E_{2}^{n(r-1) / 2} \leq C E^{(2-(n-2)(r-1)) /(2-n(r-1))}(t)+E_{2}(t),
$$

hence, we deduce that

$$
\frac{d}{d t} E_{2}(t) \leq C(t)\left(1+E_{2}(t)\right)
$$

So, we obtain

$$
E_{2}(t) \leq C^{\prime} e^{\int_{0}^{t} C(s) d s}
$$

From (2.122) and the first equation of problem (1.1), we also prove easily that

$$
\left\|\Delta_{x} u(t)\right\|_{2} \leq C^{\prime}(t)<\infty
$$

for all $t \geq 0$. Indeed, we have

$$
\left\|\Delta_{x} u(t)\right\|_{2} \leq\left\|u^{\prime \prime}(t)\right\|_{2}+\left\|\lambda^{2} u(t)\right\|_{2}+\sigma(t)\left\|g\left(u^{\prime}(t)\right)\right\|_{2}+\|f(u)\|_{2}
$$

and also we have

$$
\|f(u)\|_{2}^{2} \leq C\|u(t)\|_{2 p}^{2 p} \leq C\|u(t)\|_{n(p-1) / 2}^{2(p-1)}\left\|\Delta_{x} u(t)\right\|_{2}^{2} .
$$

Here, to check the last inequality of (2.125), we note that if $(n+4) / n \leq p \leq(n+2) /(n-2)$, then

$$
\begin{aligned}
C\|u(t)\|_{n(p-1) / 2}^{2(p-1)} & \leq C\|u(t)\|_{2}^{4-(n-2)(p-1)}\left\|\nabla_{x} u(t)\right\|_{2}^{n(p-1)-4} \\
& \leq(\tilde{d}(t))^{4-(n-2)(p-1)} E^{p-1}(t)<\frac{1}{2} .
\end{aligned}
$$


Thus the solution in the sense of Theorem 2.6 exists globally in time $t$ under the assumption $\left(u_{0}, u_{1}\right) \in H^{2} \times H^{1}$.

When $\left(u_{0}, u_{1}\right) \in H^{1} \times L^{2}$ we approximate $\left(u_{0}, u_{1}\right)$ by $\left(u_{0}^{k}, u_{1}^{k}\right) \in H^{2} \times H^{1}, k=1,2, \ldots$, in the topologies of $H^{1} \times L^{2}$, which satisfy

$$
\operatorname{supp} u_{0}^{k} \cap \operatorname{supp} u_{1}^{k} \subset\left\{x \in \mathbb{R}^{n}|| x \mid \leq L\right\} \text {. }
$$

Since $\lim _{k \rightarrow \infty}\left(u_{0}^{k}, u_{1}^{k}\right)=\left(u_{0}, u_{1}\right)$ in $H^{1} \times L^{2}$, then for these initial data problem (1.1) has global solutions $u^{k} \in W_{\text {loc }}^{2, \infty}\left([0, \infty) ; L^{2}\right) \cap W_{\text {loc }}^{1, \infty}\left([0, \infty) ; H^{1}\right) \cap L_{\text {loc }}^{\infty}\left([0, \infty) ; H^{2}\right)$, which satisfy (2.122) and (2.123). We can easily see that $\left\{u^{k}(t)\right\}$ converges uniformly on each compact interval $[0, T], T>0$. Uniqueness follow from a standard argument. The proof of Theorem 2.8 is now completed.

\section{References}

[1] A. Benaissa and S. A. Messaoudi, Blow-up of solutions for the Kirchhoff equation of q-Laplacian type with nonlinear dissipation, Colloq. Math. 94 (2002), no. 1, 103-109.

[2] A. Benaissa and L. Rahmani, Global existence and energy decay of solutions for Kirchhoff-Carrier equations with weakly nonlinear dissipation, Bull. Belg. Math. Soc. Simon Stevin 11 (2004), no. $4,547-574$.

[3] P. Brenner, On space-time means and strong global solutions of nonlinear hyperbolic equations, Math. Z. 201 (1989), no. 1, 45-55.

[4] V. Georgiev and G. Todorova, Existence of a solution of the wave equation with nonlinear damping and source terms, J. Differential Equations 109 (1994), no. 2, 295-308.

[5] J. Ginibre and G. Velo, The global Cauchy problem for the nonlinear Klein-Gordon equation, Math. Z. 189 (1985), no. 4, 487-505.

[6] A. Haraux, Two remarks on hyperbolic dissipative problems, Nonlinear Partial Differential Equations and Their Applications. Collège de France Seminar, Vol. VII (Paris, 1983-1984), Res. Notes in Math., vol. 122, Pitman, Massachusetts, 1985, pp. 161-179.

[7] R. Ikehata, Some remarks on the wave equations with nonlinear damping and source terms, Nonlinear Anal. 27 (1996), no. 10, 1165-1175.

[8] R. Ikehata and T. Suzuki, Stable and unstable sets for evolution equations of parabolic and hyperbolic type, Hiroshima Math. J. 26 (1996), no. 3, 475-491.

[9] F. John, Nonlinear Wave Equations, Formation of Singularities, University Lecture Series, vol. 2, American Mathematical Society, Rhode Island, 1990.

[10] V. Komornik, Exact Controllability and Stabilization, RAM: Research in Applied Mathematics, Masson, Paris, 1994.

[11] H. A. Levine, P. Pucci, and J. Serrin, Some remarks on global nonexistence for nonautonomous abstract evolution equations, Harmonic Analysis and Nonlinear Differential Equations (Riverside, Calif, 1995), Contemp. Math., vol. 208, American Mathematical Society, Rhode Island, 1997, pp. 253-263.

[12] H. A. Levine and J. Serrin, Global nonexistence theorems for quasilinear evolution equations with dissipation, Arch. Rational Mech. Anal. 137 (1997), no. 4, 341-361.

[13] P. Martinez, A new method to obtain decay rate estimates for dissipative systems, ESAIM Control Optim. Calc. Var. 4 (1999), 419-444.

[14] M. Nakao, A difference inequality and its application to nonlinear evolution equations, J. Math. Soc. Japan 30 (1978), no. 4, 747-762.

[15] M. Nakao and K. Ono, Existence of global solutions to the Cauchy problem for the semilinear dissipative wave equations, Math. Z. 214 (1993), no. 2, 325-342. 
[16] Global existence to the Cauchy problem of the semilinear wave equation with a nonlinear dissipation, Funkcial. Ekvac. 38 (1995), no. 3, 417-431.

[17] K. Ono, On global solutions and blow-up solutions of nonlinear Kirchhoff strings with nonlinear dissipation, J. Math. Anal. Appl. 216 (1997), no. 1, 321-342.

[18] D. H. Sattinger, On global solution of nonlinear hyperbolic equations, Arch. Rational Mech. Anal. 30 (1968), 148-172.

[19] W. A. Strauss, On continuity of functions with values in various Banach spaces, Pacific J. Math. 19 (1966), 543-551.

[20] - Nonlinear Wave Equations, CBMS Regional Conference Series in Mathematics, vol. 73, Conference Board of the Mathematical Sciences, Washington, D.C., 1989.

[21] G. Todorova, Stable and unstable sets for the Cauchy problem for a nonlinear wave equation with nonlinear damping and source terms, C. R. Acad. Sci. Paris Sér. I Math. 328 (1999), no. 2, $117-122$.

Abbès Benaissa: Département de Mathematiques, Faculté des Sciences, Université Djillali Liabès, B. P. 89 , Sidi Bel Abbès 22000, Algeria

E-mail address: benaissa_abbes@yahoo.com

Soufiane Mokeddem: Département de Mathematiques, Faculté des Sciences, Université Djillali Liabès, B. P. 89, Sidi Bel Abbès 22000, Algeria

E-mail address: mok5_so5@yahoo.fr 


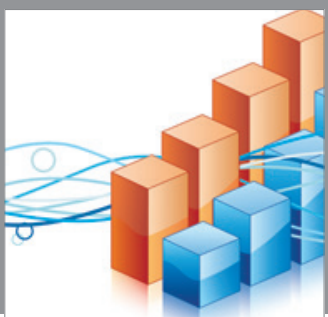

Advances in

Operations Research

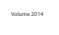

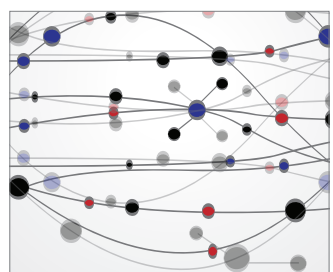

\section{The Scientific} World Journal
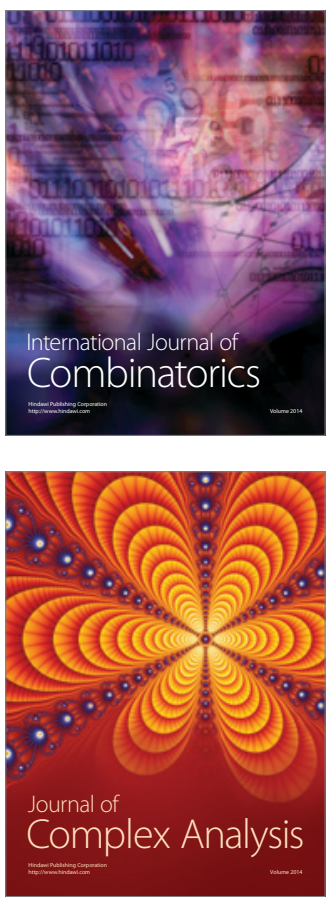

International Journal of

Mathematics and

Mathematical

Sciences
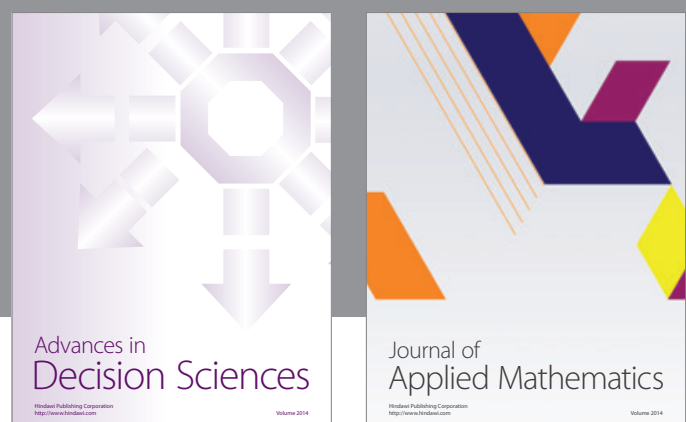

Journal of

Applied Mathematics
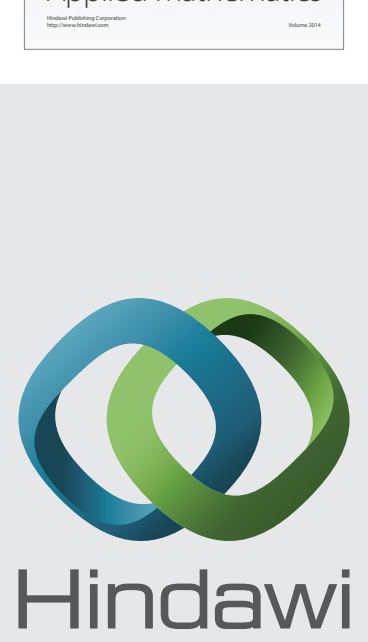

Submit your manuscripts at http://www.hindawi.com
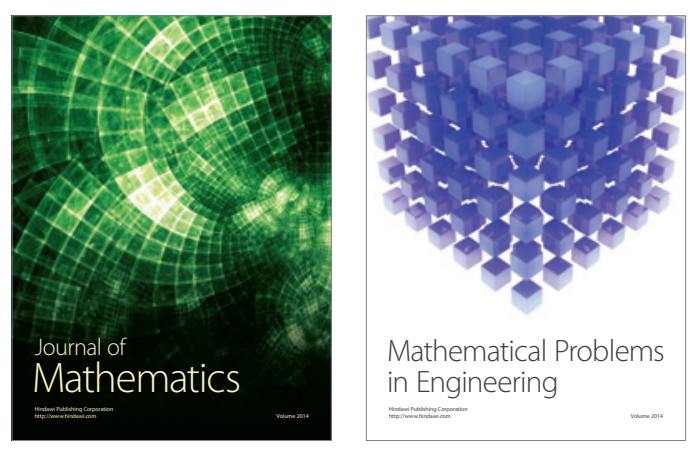

Mathematical Problems in Engineering
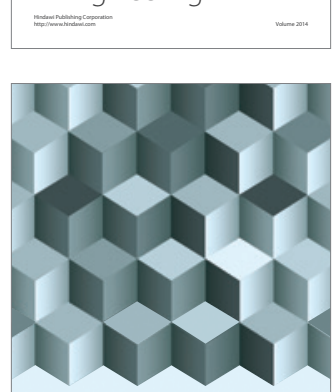

Journal of

Function Spaces
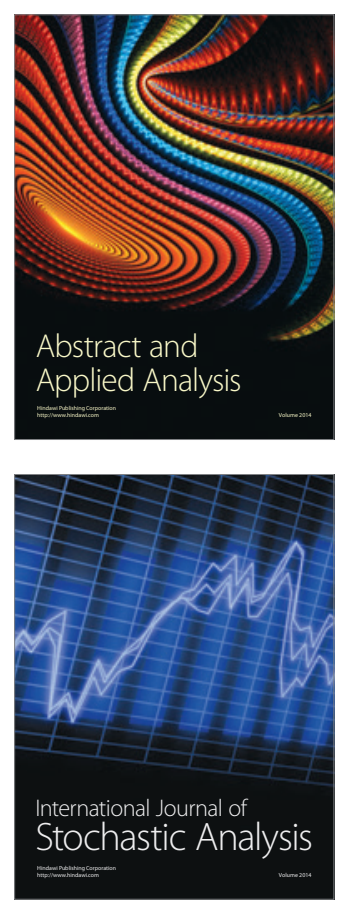

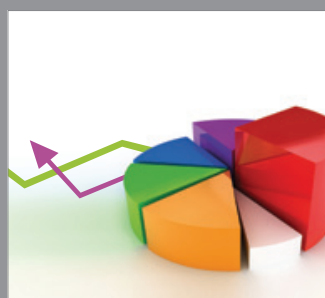

ournal of

Probability and Statistics

Promensencen
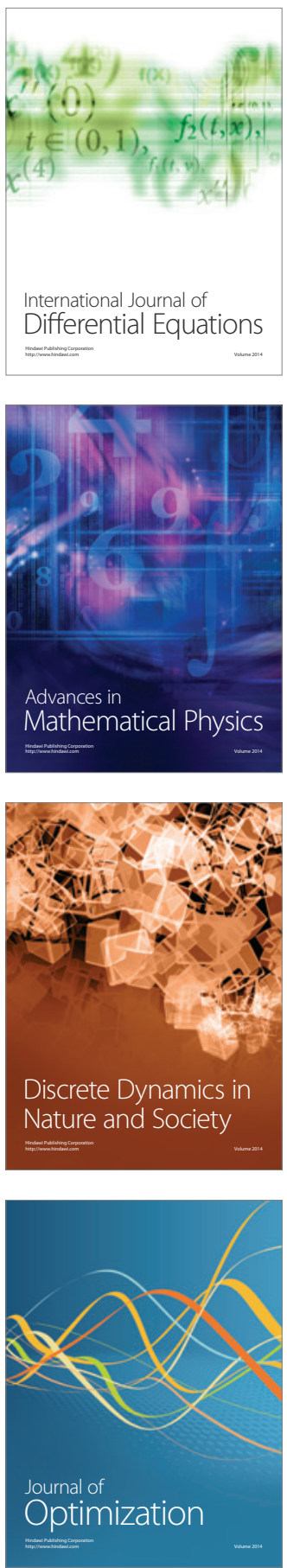